\title{
Right Coronary Artery and its Branches
}

National Cancer Institute

\section{Source}

National Cancer Institute. Right Coronary Artery and its Branches. NCI Thesaurus. Code C102340.

The right coronary artery and all of its branches, including the right posterior descending, right posterolateral and acute marginal branches. 